\title{
Ribosomal footprints
}

Translation can now be measured accurately on a genome-wide scale thanks to a novel ribosome profiling technique that uses deep sequencing to track translation with subcodon resolution. This new technique could overcome the uncertainty that is inherent in inferring protein levels from mRNA levels, owing to translation regulation.

Previous methods to measure translation directly are few and far between, and they suffer from a number of limitations, including low

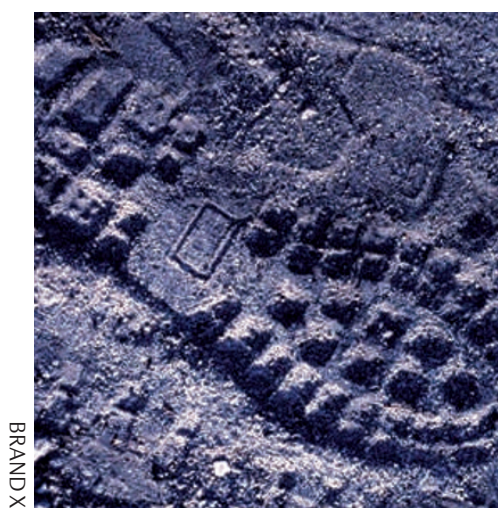

resolution. Ingolia and colleagues treated translating ribosomes in budding yeast with nucleases to produce a set of ribosome-protected mRNA fragments called footprints. They then converted these footprints - the sequences of which are indicative of the positions of active ribosomes - into ssDNA molecules through the addition of a poly (A) tail and reverse transcription, and used deep sequencing to map and quantify translation.

Sequencing 42 million footprints from yeast mRNAs gave excellent coverage of the sequences being translated - from 12-13 nucleotides upstream of the start codon to 18 nucleotides downstream of the stop codon. The sequences also showed a three-nucleotide periodicity, allowing the reading frame to be determined. By comparing the rate of translation with mRNA abundance from the same samples, the authors showed that translation efficiency can vary $\sim 100$-fold between yeast genes, and that some transcripts are translationally inactive. These findings emphasize the importance of translational regulation in the dynamics of gene expression.

The footprinting technique has given new insights into the processes of translation and translational control, including how translation responds to changing physiological conditions. For example, the authors observed increased initiation at non-AUG start codons in yeast cells under starvation conditions.

Ribosome profiling could be a valuable technique to increase our understanding of the myriad ways that cells regulate protein levels. It could also be applied to studying disease - comparisons of translational profiles would aid further characterization of abnormal cells.

Elizabeth Neame

ORIGINAL RESEARCH PAPER Ingolia, N. T. et al. Genome-wide analysis in vivo of translation with nucleotide resolution using ribosome profiling. Science 12 Feb 2009 (doi:10.1126/ science.1168978) 